\title{
Effect of Calcium Carbide on Rat Tissue
}

\section{Younus Patoare ${ }^{1}$, Md. Iqbal Hossain ${ }^{2}$, Mohammad Nurul Islam ${ }^{3}$, Akhtarunnessa Chowdhury ${ }^{4}$, Seheli Parveen ${ }^{2}$, Md Mahboob Hossain ${ }^{1}$ and Abul Hasnat ${ }^{2}$}

\author{
${ }^{1}$ Department of Pharmacy, University of Asia Pacific, Dhanmondi, Dhaka-125, Bangladesh \\ ${ }^{2}$ Department of Clinical Pharmacy \& Pharmacology, Dhaka University, Dhaka-1000, Bangladesh \\ ${ }^{3}$ Department of Botany, University of Dhaka, Dhaka-1000, Bangladesh \\ ${ }^{4}$ Department of Zoology, University of Dhaka, Dhaka-1000, Bangladesh
}

\begin{abstract}
To evaluate the effects of Calcium carbide $\left(\mathrm{CaC}_{2}\right)$ in biological system, an in vivo study was carried out on Long Evans rats. $\mathrm{CaC}_{2}$ was administered orally once daily for one month with specific concentrations. GroupI was considered as the control group (without $\mathrm{CaC}_{2}$ ), Group - II, III, IV and V were the sample groups treated with $\mathrm{CaC}_{2}$ having concentration of $1 \mathrm{~g} / \mathrm{kg}, 2 \mathrm{~g} / \mathrm{kg}, 5 \mathrm{~g} / \mathrm{kg}$ and $10 \mathrm{~g} / \mathrm{kg}$ body weight respectively. The experiment was conducted to detect any cellular and molecular level changes caused by $\mathrm{CaC}_{2}$. The histopathology and isozyme assay were performed to analyze the changes in the activities of the genes affected by the free radicals released from $\mathrm{CaC}_{2}$. The molecular analysis included different isozymes namely esterase and acid phosphatase. Polyacrylamide electrophoresis of whole cell extract of control subjects and $\mathrm{CaC}_{2}$ administered rats were performed; subsequently the gels were treated with the substrates for acid phosphatase and esterase respectively. No difference was observed in the whole cell extract band pattern between the control subject and the $\mathrm{CaC}_{2}$ administered rats, which grossly indicates that the $\mathrm{CaC}_{2}$ has no effect on the expression pattern of isozymes (acid phosphatase and esterase). Histopathological analysis of liver, heart, spleen, kidney and lungs were performed to observe any change due to the administration of $\mathrm{CaC}_{2}$. Remarkable changes were observed during the histopathological study of lungs and kidney only. The histopathological analysis of kidney showed the thickening of the lining of collecting tubules with changes in cell structure while lungs were found to be increased moderately in weight, with focal areas of consolidation that was found red-brown to red.
\end{abstract}

Key words: Calcium carbide, Histopathological study, Isozymes

\section{INTRODUCTION}

Calcium carbide is a chemical compound with the formula $\mathrm{CaC}_{2}$. Its main use now is as a source of acetylene. It is a toxic substance banned under the Prevention of Food Adulteration Act. Calcium carbide contains traces of hazardous arsenic and phosphorous. Once dissolved in water, the carbide produces acetylene gas that quickens the ripening process, hence is used in ripening fruits. ${ }^{1}$ In human

Correspondence to: Abul Hasnat

Email- ahasnat99@yahoo.com

Tel. 02-8612069; Fax-02-8615583

Dhaka Univ. J. Pharm. Sci. 6(2): 93-98, 2007 (December) after ingestion acetylene produces free radicals which initiate detrimental effects on various organs. ${ }^{2}$ Scientists now know that free radicals play a major role in the aging process as well as in the onset of cancer, heart disease, stroke, arthritis, and possibly allergies and a host of other ailments. ${ }^{3}$ Oxidative DNA damage from active oxygen species such as hydroxyl radical (HO•) has been hypothesized to play a critical role in diverse biological processes including mutagenesis, carcinogenesis, radiation damage and cancer chemotherapy. ${ }^{4}$

A free radical is a cell killer that wreaks havoc by damaging DNA, altering biochemical compounds, 
corroding cell membranes, and destroying cells outright. $^{3}$ By definition a free radical is any atom (e.g. oxygen, nitrogen) with at least one unpaired electron in the outermost shell, and is capable of independent existence. ${ }^{5}$ A free radical is easily formed when a covalent bond between entities is broken and one electron remains with each newly formed atom. ${ }^{5}$ Free radicals are highly reactive due to the presence of unpaired electron(s).

The most recent studies reveal that calcium carbide is vigorously used in fruits ripening (e.g. mango) in the third world countries, especially in India, Indonesia and Bangladesh. The ripening signals of a fruit come from ethylene. Production of ethylene turns on some genes that are transcribed and translated to produce other enzymes. ${ }^{6}$ These enzymes are responsible for the conversion of starch into simple sugar, degradation of chlorophyll and appearance of other new pigments like carotenoids, change in the skin colour and the breakdown of acid, making the fruit taste neutral. ${ }^{6}$ For a number of years, some fruit vendors in West Africa have used calcium carbide to ripe immature fruits before sale. This permits the growers to harvest fruits before maturity to get money sooner, which is harmful for human being. It is impossible to conduct the clinical trial in human being due to toxic effect of calcium carbide as well as it is banned ethically to do any clinical trial on humans. That's why the experiment was performed in rats to observe any harmful effect of calcium carbide. This experiment was conducted to investigate the amount and concentration of calcium carbide harmful for human body. Molecular analysis is very important for this investigation because $\mathrm{CaC}_{2}$ forms free radicals, which may interact with the structure of DNA and may change the expression level of gene. That's why we wanted to observe the activity of isozymes of esterase and acid phosphatase from different organs like lungs, heart, liver, spleen and kidney of rat treated with $\mathrm{CaC}_{2}$ and control rats. To know this activity we used polyacrylamide gel electrophoresis method (PAGE). The cellular analysis is also important for this experiment, which was performed in BCSIR and Zoology Department of
Dhaka University. From this cellular analysis we observed the effect of calcium carbide on cellular structure of different organs.

\section{MATERIALS AND METHODS}

A total of 25 Long Evans rats of either sex, weighting 65-150 gm were used to perform the study. They were purchased from the animal resource branch of ICDDR,B. The animals were kept in standard environmental condition, given standard rodent food (ICDDR,B formulated) and water ad libitum. The rats were divided into five groups based on the body weight and then different concentrations of calcium carbide were administered to the rats of various groups. Each group composed of five rats. Rats with average weight of 150 gm were considered as the control group and marked as group-I; the other five groups were considered as sample groups and marked as group-II, group-III, group-IV and groupV. A concentration of $1 \mathrm{~g}, 2 \mathrm{~g}, 5 \mathrm{~g}$ and $10 \mathrm{~g} / \mathrm{kg}$ body weight of calcium carbide was administered orally once daily for one month to sample groups, i.e. group-II, group-III, group-IV and group-V. Group-I (control group) was supplied with normal foods and no drug was administered. After administration of calcium carbide for one month, different important organs (liver, heart, kidney, spleen and lungs) were collected from the rats of both control and sample groups to investigate the effect of calcium carbide. Two types of experiments, such as histopathology of liver, kidney, heart, lungs and spleen and isozyme assay (esterase and acid phosphatase) were conducted.

Histopathology of liver, Kidney, Heart, Lung and Spleen. Histopathological studies of liver, kidney, heart, lungs and spleen were performed to observe any change in the cellular structures (degeneration or regeneration) of the rats receiving calcium carbide at a different dose for 30 days with respect to control group.

\section{Procedure}

Collection and processing of the tissue. The liver, kidney, heart, lung and spleen were collected 
from rats of sample groups and control group following sacrificing them after one-month. The tissues were sliced into pieces each measuring a few millimeter in thickness. The sliced tissues were then immersed in 10\% formalin for 24 hours. The tissues were dehydrated in ascending order of absolute alcohol and embedded in paraffin. The blocks were sectioned with the help of rotating microtone at 6micron thickness.

Staining. The sections were deparaffinized twice with xylene (5 mins, in each) and hydrated in descending order of alcohol (2-3 mins, in each). The sections were kept in Erchlich's hematoxylin after washing with water. Finally they were washed in running water. Counter staining in Eosin was done (2-3 minutes) and the sections were dehydrated in ascending order of absolute alcohol (2-3 minutes). The sections were then cleaned twice with xylene (5 minutes).
Mounting. Glass slides containing the tissues area were wiped dried and then a drop of canada balsam was put on the section and the coverslip was gently placed on it. On the sections, thin film between the coverslip and the slide with the mounting medium (canada balsam) was performed to attach them. Histopathological examinations were done under high power microscope and were recorded by photographs. Then slides of sample groups were compared with the slides of control group.

Molecular analysis: Isozyme assay. The isozyme systems, namely acid phosphatase and esterase were used in the present investigation. These experiments were carried out according to the method of Arus and Orton. ${ }^{7}$ Polyacrylamide gel electrophoresis (Vertical; SDS-PAGE) was carried for the investigation of the cited isozymes. Two separate methods of staining were used to detect the enzyme activity on the gels.

\section{Material}

\begin{tabular}{lll}
\hline Separating gel & Stacking gel & Sample buffer \\
\hline $10 \%$ acrylamide solution & $10 \%$ acrylamide & $75 \%$ glycerol \\
Tris (pH 8.8), & Tris (pH 6.8) & $25 \%$ Bromophenol blue. \\
Water, & Glycerol & Tris-HCl \\
$10 \%$ Ammonium persulfate (APS) & Water & SDS \\
Tetramethyl ethylene diamine (TEMED) & $10 \%$ Ammonium persulfate (APS) & \\
& Tetramethyl ethylene diamine (TEMED) & \\
\hline
\end{tabular}

Top electrode buffer (pH 8.3); Lower electrode buffer (pH 8.9)

Before loading, the samples were diluted at a ratio of 2:1 with the sample buffer. Samples containing 100 and $150 \mu \mathrm{g}$ of proteins were applied for running the gels. After running, the gels were stained by different methods for two different types of enzyme localization. Following incubation mixtures were used for the detection of two different isozymes.

Isolation of protein from different organs. Firstly the samples were crush in cold condition then phosphate buffer was added with this crushed sample. Samples were centrifuged at $4^{\circ} \mathrm{C}$ with the maximum speed $(15,000 \mathrm{rpm})$ and Finally the supernatant was collected from this sample. Protein concentration was determined by Lowry method. ${ }^{8}$
SDS-PAGE method. Separating gel was first prepared using the material stated in the above table. Then stacking gel was prepared and placed at the top of the separating gel. Protein mixture of control and sample groups were prepared with help of sample buffer and stored separately in different tubes. Tris (pH 6.8) was used to remove low molecular weight impurities prior to addition of protein mixtures. Glycerol was added to increase the sample density, facilitating gel loading and preventing convective migration out of the sample wells. A small amount of bromophenol blue was added as a visual aid during sample loading and as a tracking dye, allowing easy monitoring of electrophoretic progress. Protein samples were loaded in individual wells at the top of polyacrylamide gel. An electric field across the gel 
was applied which drove negatively charged polypeptides to the anode. Stain was used to figure out changes between proteins of sample and control groups.

\section{RESULTS AND DISCUSSION}

Histopathology of liver, kidney, heart, lung and spleen. Histopathology of liver, kidney, heart, lung and spleen were performed to observe any change in the cellular structures (degeneration or regeneration) of the rats receiving the calcium carbide at a different dose for 30 days with respect to control group. Slides for histopathological analysis were examined. The photographs clearly showed remarkable changes in organs of rats treated with calcium carbide compared to the organs of control group (Figures 1 \& 3).

Neither morphological nor structural changes were observed in case of heart, spleen and liver in rats treated with $\mathrm{CaC}_{2}$. Marked changes were observed in case of lungs and kidney only. The calcium carbide treated lungs were moderately increased in weight, with focal areas of consolidation that was found red-brown to red (Figure 2). Histopathologically, fibrous thickening of septa and hypertrophy of lining septal cell were observed in rats of sample group. Histopathological analysis of kidney showed thickening of the lining of the collecting tubules with change in cell structure and also revealed some glomerulus structure in raptured condition (Figure 4).
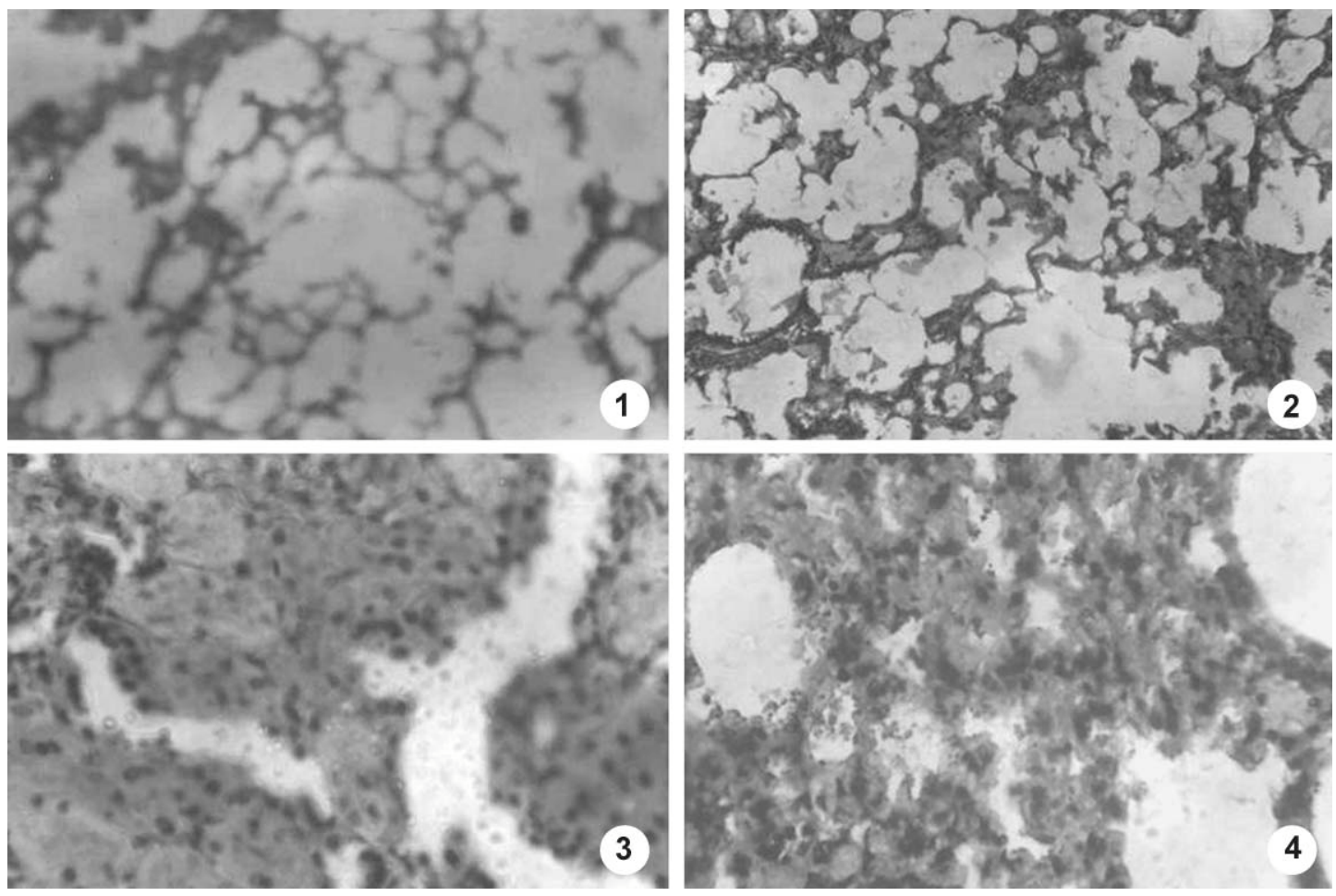

Figures 1-4. 1. Cross section of lungs (control group). 2. Cross section of lungs (group-5). 3. Cross section of kidney (control group). 4. Cross section of Kidney (Group-5)

\section{Molecular analysis: Isozyme assay}

Enzyme localization. Activity of isozymes of esterase and acid phosphatase from organs of rat with and $\mathrm{CaC}_{2}$ was studied following vertical polyacrylamide gel electrophoresis. The electrophoresis gels were stained for different isozymes. When the bands appeared they were zymogramed immediately. The enzyme activities in 
terms of the intensity of the bands were recorded as light, dark and very dark. The activity of these enzymes detected on polyacrylamide gels is found as follows:

Esterase. Esterase activity on the polyacrylamide gel produced by various organs of rats either untreated or treated with $\mathrm{CaC}_{2}$ was observed. A number of bands of same intensities were developed in the different groups of sample, which was treated with $\mathrm{CaC}_{2}$ compared to untreated $\mathrm{CaC}_{2}$ (control group). These organs showed homogeneity regarding their pattern and each of the groups of organs possessed unique banding profile.
Acid phosphatase. As the protein band intensity for different organs were found similar in case of esterase, the experiments with acid phosphatase was done only in heart tissue of both treatment and control group. Like esterase, after staining with substrates for acid phosphatase, bands of same intensities were observed in different groups of sample, which was treated with $\mathrm{CaC}_{2}$ compared to untreated one (Figure 5). In fact no difference was observed in the whole cell extract band pattern between the control subject and the $\mathrm{CaC}_{2}$ administered rat, which grossly indicates that the $\mathrm{CaC}_{2}$ has no effect on the level of expression of isozymes (acid phosphatase and esterase).

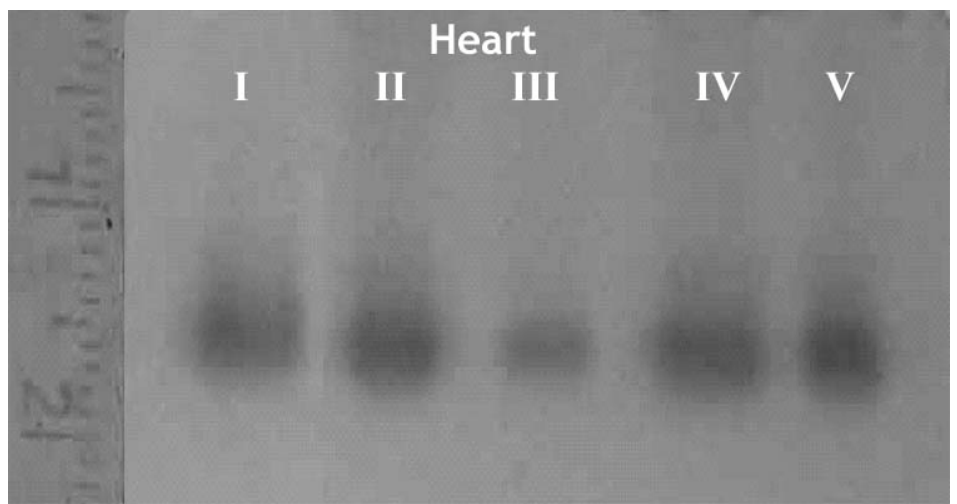

(a)

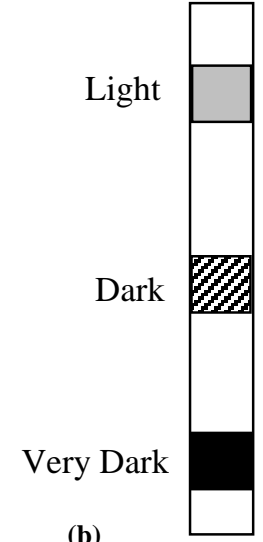

(b)

Figure 5: a. Acid phosphatase activity on the polyacrylamide gel using heart tissue of group-V. b. A zymogarm shows the group of I, II, III, $\mathrm{IV}$ and $\mathrm{V}$ because of the same band of intensity.

Calcium Carbide, a vigorously used fruitripening agent in the $3^{\text {rd }}$ world countries, shows toxic effects on biological system. Chemically $\mathrm{CaC}_{2}$ releases free radical due to chemical degradation, which is very harmful for any biological system. The free radicals released from $\mathrm{CaC}_{2}$ have a probability to interact with the gene and to change the basic information of DNA code resulting in cancer. $^{9}$ Present histopathological study illustrated detrimental effects of calcium carbide on lungs and kidney of the experimented rats. Numerous epidemiologic evidences on the relationship between calcium carbide exposure and cancer were reviewed earlier. ${ }^{10}$ Heavy exposure to the chemical entailed a substantial risk of lung, skin, and bladder cancer according to those studies. ${ }^{11}$ These findings are in agreement with the study of Kjuus et al. ${ }^{10}$ who found calcium carbide exposure produced liver and nephrotoxicity along with colonic and prostatic cancer. ${ }^{10}$ Similar results were also found from the study of Vecchia et al. ${ }^{12}$

The development of cancer is generally divided into three stages: initiation, promotion, and progression. ${ }^{13}$ Reactive oxygen species (ROS), oxygen containing molecules that have a higher reactivity than ground-state oxygen $\left(\mathrm{O}_{2}\right)$, and free radicals, molecules that contain at least one unpaired electron, are postulated to be involved in this process, especially in the stage of initiation and promotion. ${ }^{13}$ The free radicals produced from calcium carbide may damage the cell in such process. 
The molecular analysis included two different isozymes namely esterase and acid phosphatase. No difference was observed in the whole cell extract band pattern between the control subject and the $\mathrm{CaC}_{2}$ administered rats, which grossly indicates that the $\mathrm{CaC}_{2}$ may have no effect on the degradation capacity or in the expression level of isozymes (acid phosphatase and esterase). Thus, from the above results and discussions, we may conclude that $\mathrm{CaC}_{2}$ may have toxic effects to the rat cells but has no effect on isozymes. To know the precise effect of $\mathrm{CaC}_{2}$ on isozymes, the protein needs to be purified and isozyme marker should be used. Probably a higher concentration with longer duration of study can exhibit the true picture of toxicity of $\mathrm{CaC}_{2}$ to the live cells.

\section{REFERENCES}

1. Halliwell, B. and Gutteridge, J.M.C. 1985. The chemistry of oxygen radicals and other oxygen-derived species. In: Free Radicals in Biology and Medicine. New York: Oxford University Press. 20-64.

2. Kaczmarski, M. J., Wojicicki, L., Samochowiee, T., Dutkiewicz, and Sych, Z. 1999. The influence of exogenous antioxidants and physical exercise on some parameters associated with production and removal of free radicals. Pharmazie 54, 303-306.
3. Del Mastero, R.F. 1980. An approach to free radicals in medicine and biology. Acta. Phyiol. Scand. 492, 153-168.

4. Fang, Y.Z., Yang, S. and Wu GY. 2002. Free radicals, antioxidants, and nutrition. Nutrition. 18, 872-879.

5. Karlsson, J. 1997. Exercise, muscle metabolism and the antioxidant defense. World Rev. Nutr. Diet. 82: 81-100.

6. Mariappan, J.J. 2002. College of Arts and Science, Pudukkottai, Tamil Nadu. 2002.

7. Arus, P. and Orton, T.J. 1983. Inheritance and linkage relationships of isozyme loci in Brassica oleracea. J. Hered. 74(6), 405-412

8. Lowry, O.H., Rosebrough, N.J., Farr, A.L. and Randall, R.J. 1951. Protein measurement with the folin phenol reagent. $J$. Biol. Chem. 193, 265-275.

9. Chen, Q. A., Fischer, J.D. 1995. Oxidative DNA damage and senescence of human diploid fibroblast cell. Proc. Natl. Acad. Sci. 92, 4337-4341.

10. Kjuus, H., Andersen, A. and Langård, S. 1986. Incidence of cancer among workers producing calcium carbide. $\mathrm{Br}$.J. Ind. Med. 43(4), 237-42.

11. Jourenkova, N. and Gustavsson, P. 1997. Cancer risk from occupational and environmental exposure to polycyclic aromatic hydrocarbons. Cancer Causes Control. 8(3): 444-72

12. La Vecchia, C. and Bosetti, C. 2003. Cancer risk in carbon electrode workers: an overview of epidemiological evidence. Eur. J. Cancer. Prev. 12(5), 431-444.

13. Li, S. J., Yan, T., Yang, J.Q., Oberley, T.D. and Oberley, L.W. 2000. The role of cellular clutathine peroxidase redox regulation in suppression of tumor cell growth by manganese superoxide dismutase. Cancer Res. 60, 3927-3939. 